\title{
Análisis del proceso de ablandamiento de trocería de encinos para la producción de chapa para madera contrachapada (triplay)
}

\author{
David Zavala Zavala ${ }^{1}$ \\ Martín Lara Rubio ${ }^{2}$
}

\section{RESUMEN}

Un uso potencial de los encinos es la producción de madera contrachapada (triplay), para lo cual se requiere definir las condiciones más adecuadas del calentamiento de la madera, del rebanado o torneado de las trozas, del secado de la chapa y del ensamble del tablero. En este trabajo se determinaron los parámetros óptimos del proceso de ablandamiento de la madera para la producción de chapa para madera contrachapada utilizando dos trozas por especie de: Quercus candicans, Quercus laurina y Quercus rugosa. De estas trozas se generaron los Aflitches@o cuadrados, que se calentaron en un tanque metálico, a través de serpentines con aceite térmico y usando agua como medio termoconductor a la madera. En cada cuadrado se insertaron dos termopares para medir el gradiente de temperatura durante el calentamiento. Dos de los cuadrados $(Q$. candicans y $Q$. laurina), se procesaron a $60{ }^{\circ} \mathrm{C}$, otros dos ( $Q$. candicans y $Q$. rugosa) a $65^{\circ} \mathrm{C}$ y los dos últimos (Q. laurina y $Q$. rugosa) a $70^{\circ} \mathrm{C}$. En general, la chapa de $Q$. candicans y $Q$. laurina, fue de mejor calidad en cuanto a textura, grietas pequeñas y uniformidad de coloración. La chapa de $Q$. rugosa presentó variación en la coloración, con manchas café-oscuras, seguramente por el ataque de bacterias al arbolado, además de pequeñas perforaciones en las chapas generadas en la parte más externa de la troza, debido a grandes dimensiones de los rayos. Se considera que la temperatura de corte en el rango de 60 a $75^{\circ} \mathrm{C}$, es adecuada para procesar encinos. En cuanto al tiempo de calentamiento utilizado en este estudio, de 50 a 65 horas, se recomienda usarlo como referencia, por considerar que las prácticas y equipos de ablandamiento cambian de una fábrica a otra. Respecto al veteado de la chapa fue muy uniforme en su estructura, aún cuando $Q$. candicans y $Q$. laurina son de porosidad circular; esta característica no se manifestó en el veteado de Acatedral@que es el más demandado por el usuario final de madera contrachapada.

\section{PALABRAS CLAVE:}

Industria forestal, ablandamiento de la madera, madera contrachapada (triplay), chapa, encinos.

\section{ABSTRACT}

A potential utilization of oak wood is the production of plywood, but it is necessary to define the more adequate conditions for the heating of wood, for the slicing or peeling of the logs, for the drying of veneer and assembling of the plywood. In this study the optimum parameters for the softening of wood for the production of plywood were determined using two logs of each one of the following species: Quercus candicans, $Q$. laurina and $Q$. rugosa. From

1 Centro Nacional de Investigación Disciplinaria en Conservación y Mejoramiento de Ecosistemas Forestales.INIFAP. Progreso No. 5. Col. Carmen Coyoacán. México, D.F. C.P. 04110 México.

2 Centro de Investigación Regional-PACIFICO CENTRO.INIFAP

Manuscrito recibido para su publicación el 12 de Septiembre de 1997 
these logs, flitches were obtained, which were heated in a metallic container through coils with thermic oil to heat water up used as thermoconducting medium for the wood. In each flitch, two thermocouples were embedded to mesure the temperature gradient during the heating. Two of the flitches $(Q$. candicans and $Q$. laurina), were tested at $60^{\circ} \mathrm{C}$, other two (Q. candicans and $Q$. rugosa) at $65^{\circ} \mathrm{C}$ and the last two $(Q$. laurina and $Q$. rugosa) at $70^{\circ} \mathrm{C}$. In general the veneer of $Q$. candicans and $Q$. laurina, was the best in texture, with small checks and uniform in color. The veneer of $Q$. rugosa showed some variation in color, with dark-brownish spots, apparently due to bacterial attack to the trees, and small holes in the veneer from the outer part of the log, due to large rays dimensions. The temperature between 60 to $75^{\circ} \mathrm{C}$ is considered adequate to process oak wood. Regarding the heating time used in this study, form 50 to 65 hours, it is suggested to use it as a guideline due to the changes in the practices and equipment between plywood mills. Regarding the porous arrangement in the veneer, it was very uniform in its structure, even though $Q$. candicans and $Q$. laurina have circular porous distribution; this characteristic was not reflected in the Acathedral@ configuration, which has a higher demand by the final user of plywood.

\section{KEY WORDS:}

Forest industry, softening of wood, plywood, veneer, oak wood.

\section{INTRODUCCION}

En los bosques de clima templado, después de las coníferas, las especies más abundantes son los encinos. No obstante su importancia en cuanto a existencias maderables, los volúmenes que se aprovechan son muy reducidos, en parte por desconocimiento de sus características tecnológicas y por la baja rentabilidad de los procesos de industrialización. Se les considera en general como especies subutilizadas, aprovechándose en cantidades reducidas para la elaboración de mangos para herramientas, para implementos agrícolas, para duela, para lambrín y para parquet; todos estos productos con ventajas tecnológicas en comparación con otras especies usadas para el mismo fin. Una mayor proporción de los volúmenes aprovechados de encinos se destina para leña, para la elaboración de carbón, como astillas para material celulósico y para la producción de tableros aglomerados. Este tipo de aprovechamiento, aún cuando puede no ser el más adecuado desde el punto de vista técnico y económico, es más aceptable que la práctica de cinchamiento del arbolado, sin ningún aprovechamiento de la madera y que se aplica únicamente para cubrir el requisito de "autorización de permisos de aprovechamiento" en bosques mezclados de pino-encino, establecido por las normas oficiales para eliminar o contrarrestar los volúmenes de encinos en relación a los de pino. Se considera que el aprovechamiento ideal es utilizar este recurso definiendo el proceso más adecuado acorde a las características de la trocería, a las especies y a la rentabilidad de los procesos.

Un uso potencial de los encinos es la producción de chapa para madera contrachapada (triplay), que generalmente se importa para abastecer la demanda nacional. Con la finalidad de aprovechar los recursos del país para este tipo de productos, se han tenido experiencias aisladas sobre la utilización de los encinos para chapa, que se han abandonado por problemas relacionados con una selección de especies, por la mala calidad de la trocería utilizada, por deficiencias del proceso de industrialización y por la carencia de un veteado vistoso, con la tendencia a compararlo con el veteado de los encinos americanos. El veteado de Acatedral@que es el más atractivo y que busca el consumidor, lo genera en gran 
parte la porosidad circular, de especies seleccionadas y que generalmente ya son cultivadas en los países donde la utilización de los encinos se orienta para productos de calidad y de alto valor económico.

La producción de chapa con encinos mexicanos se considera factible si se hace una selección de las especies con base en sus características tecnológicas, en su abundancia, en la conformación del arbolado y en la adopción de técnicas adecuadas de los procesos de ablandamiento de la trocería, del rebanado o torneado de la madera, del secado de la chapa y de la elaboración de madera contrachapada; con lo cual, además de sustituir importaciones de trocería, de chapa y de madera contrachapada, se tendría la posibilidad de exportar estos productos, puesto que la demanda existe a nivel internacional.

\section{OBJETIVOS}

La finalidad de este trabajo fue determinar los parámetros óptimos de procesamiento para la producción de chapa para madera contrachapada de tres especies de encinos, $Q$. candicans, $Q$. laurina y $Q$. rugosa; analizando el proceso de calentamiento o acondicionamiento de la madera, el proceso de rebanado y el secado de la chapa e interrelacionando las propiedades tecnológicas de la madera con las variables del proceso.

\section{ANTECEDENTES}

Utilización de los encinos mexicanos para chapa. De los trabajos realizados en México sobre la utilización de encinos para chapa, se tiene el de Voirol (1985), que señala las características de los encinos potencialmente utilizables para la producción de chapa: fuste recto, duramen sano, grano recto, baja densidad, rayos pequeños y poros distribuidos en bandas circulares para lograr una figura decorativa. Ejemplifica que algunos de los encinos con estas características podrían ser: $Q$. conspersa, $Q$. laurina y $Q$. acutifolia. Señala la necesidad de calentar las trozas previamente al torneado en un rango de 60 a $90^{\circ} \mathrm{C}$ y realizar el calentamiento a una velocidad de $4^{\circ} \mathrm{C}$ por hora. Entre las fases del proceso de producción de chapa de encinos, destaca el ablandamiento de la trocería por medio de agua caliente en pilas. A este respecto, Zavala (1991) encontró que la industria de madera contrachapada de la zona metropolitana de la Ciudad de México, las temperaturas de las trozas al momento del corte varían entre industrias, aún cuando se trate de la misma especie, presentándose un rango de 25 a $45^{\circ}$ C. Zavala y Trujillo (1993), determinaron la temperatura óptima de corte para obtener chapa de buena calidad de madera de encinos americanos procesados en la fábrica de "Chapas los Reyes", siendo de $68^{\circ} \mathrm{C}$ al momento de sacar la troza de las pilas de acondicionamiento y de $47^{\circ} \mathrm{C}$ la temperatura de la chapa generada.

Entre otras experiencias de industriales (Andrade y Alvarez, 1997), se tienen los intentos que se han realizado en la fábrica "Enchapados Alfa", en Durango y en la fábrica de "Chapas los Reyes", en el Edo de México. En el primer caso, se logró producir chapa y madera contrachapada de algunos encinos del Estado. de Durango hace aproximadamente 5 años, dejando de utilizarlos por la dificultad del proceso; debido en parte a la mezcla de especies con características muy diferentes que repercutían en la calidad de la chapa y por falta de mercado del producto final. En el caso de AChapas Los Reyes@ un argumento sobre la reducida utilización de los encinos mexicanos es la diferencia de veteado y la mayor dificultad en el proceso de transformación, comparado con los encinos americanos, lo cual limita la demanda de la chapa y de madera contrachapada y la posibilidad de 
industrialización con márgenes económicos atractivos.

Respecto a las características de la madera para chapa, aún cuando no se han determinado experimentalmente, De la Paz (1976), sugiere las especies: Quercus acutifolia y $Q$. candicans para chapa fina y $Q$. crispipilis y $Q$. scytophylla los recomienda para chapa en general (los cuatro son encinos rojos).

De la Paz y Aguilar (1978), señalan las diferencias anatómicas entre los encinos rojos y blancos, específicamente en cuanto al color, al tamaño y grosor de los rayos poliseriados, al contenido de vasos y a la abundancia de cristales. Con base en estas diferencias generalizan que los encinos blancos se utilizan para barricas de añejamiento, armazones para construcción, cajas de empaque y embalaje, durmientes, postes para cercas y pilotes para minas. Los encinos rojos se usan para pisos, lambrines, chapa, muebles, cabos y mangos para herramientas, implementos agrícolas, utensilios domésticos, hormas para calzado, tarimas para carga, partes para instrumentos musicales y para la elaboración de juguetes.

Proceso de acondicionamiento de la madera. En el proceso de corte de las trozas o cuadrados influyen varios factores determinantes de la calidad de la chapa, destacando la temperatura y el contenido de humedad de la madera al momento del torneado o rebanado. La temperatura de corte de la madera generalmente se logra sometiéndola a un proceso de calentamiento, cuya eficiencia depende del medio termoconductor empleado y de las características de las especies procesadas, destacando el diámetro o dimensiones de las trozas, la densidad y el contenido de humedad de la madera (Lutz et al., 1967; Lutz y Patzer, 1976; Walser 1974).
Ventajas del calentamiento. El proceso de ablandamiento o calentamiento de la madera previamente a su corte para la producción de chapa, se considera necesario para especies duras o de alta densidad, de grano irregular o con veteado pronunciado, en maderas resinosas donde puede interferir la resina con otras fases de preparación de la chapa y producción del tablero y cuando se desea producir chapa con espesores mayores de $3.2 \mathrm{~mm}(1 / 8$ plg), que de no estar calentada se generarían grietas pronunciadas y pérdidas excesivas de material (Fleischer, 1959; Lutz 1974).

Temperatura de la madera durante el corte o rebanado de la chapa. La temperatura de la madera al momento del corte determina la calidad de la chapa respecto a su tersura, rajaduras, grietas y vellosidades, además de la facilidad o dificultad del corte. Estos factores reflejan la importancia de precisar el rango óptimo de temperaturas de la madera previamente a su procesamiento. Se considera en general que las maderas suaves (peso específico menor de $0.40 \mathrm{~g} / \mathrm{cm}^{3}$ ), no requieren de calentamiento para obtener chapa de buena calidad. (Palka, 1974; Peters, 1974; Feihl y Godin, 1975; Lutz, 1978).

Fleischer (1959), señala que en el caso de hojosas, la temperatura de corte esta muy ligada con la densidad de la madera y establece que para especies de 0.46 a $0.55 \mathrm{~g} / \mathrm{cm}^{3}$, la temperatura de corte es de $59^{\circ} \mathrm{C}\left(140^{\circ} \mathrm{F}\right)$, para las de 0.55 a $0.59 \mathrm{~g} / \mathrm{cm}^{3}$ de $70^{\circ} \mathrm{C}\left(160^{\circ} \mathrm{F}\right)$ y para las de $0.60 \mathrm{a} 0.64 \mathrm{~g} / \mathrm{cm}^{3}$ de $92^{\circ} \mathrm{C}\left(200^{\circ} \mathrm{F}\right)$. Indica que estas temperaturas se aplican en maderas de hilo recto y en corte rotatorio y que para chapa rebanada las temperaturas requeridas son de 6 a $11^{\circ} \mathrm{C}\left(10\right.$ a $\left.20^{\circ} \mathrm{F}\right)$ más altas. Analizando información generada por Lutz (1972), indica que la mayoría de los encinos se tornean a una temperatura de 59 a $70^{\circ} \mathrm{C}\left(140\right.$ a $\left.160^{\circ} \mathrm{F}\right)$ y se rebanan de 81 a $92^{\circ} \mathrm{C}\left(180\right.$ a $\left.200^{\circ} \mathrm{F}\right)$. 
Entre otros estudios que proporcionan información sobre las temperaturas de corte de la madera, se tiene el de Palka (1974), que señala que para la mayoría de las especies, las temperaturas requeridas en la madera al momento del corte de la chapa, varían de 38 a $71^{\circ} \mathrm{C}$. El de Moreno y Espejel (1983), que indican que el rango de temperaturas observado en las trozas al momento del corte en torno, ha sido de 43 a $52^{\circ} \mathrm{C}$ en el centro y de 65 a $70^{\circ} \mathrm{C}$ en la periferia. El anteriormente citado de Zavala (1991), quien señala que las temperaturas en las trozas al momento del corte varían en un rango de 25 a $45^{\circ} \mathrm{C}$.

\section{Efecto de la densidad de la madera en el} tiempo de acondicionamiento. La densidad de la madera es uno de los factores determinantes del tiempo de calentamiento para su acondicionamiento y posterior rebanado o desenrollado. Feihl (1971) y Feihl y Godin (1975), indican que se requiere más tiempo para calentar una troza de alta densidad que una de baja densidad, estableciendo que la madera de diferentes especies con densidad anhidra similar, pero con densidad verde diferente, las de mayor densidad verde requieren de mayores tiempos de calentamiento. Con base en sus experimentos encontraron que las trozas de alta densidad requieren $25 \%$ más tiempo de calentamiento que las de densidad media, en tanto que las de densidad baja requieren un $25 \%$ de menos tiempo de calentamiento, con relación a las de densidad media.

Fleischer (1959), establece que la velocidad de calentamiento varía inversamente con el peso específico y que la diferencia de especies, indistinta del peso específico, es insignificante. Adicionalmente, presenta gráficas para determinar la temperatura de corte en función de la densidad de la madera y establece la relación entre estos dos factores con base en la difusibilidad térmica que cambia con la densidad.
Zavala (1991), presenta los tiempos de calentamiento de especies con diferentes densidades utilizados en las industrias de madera contrachapada de la zona metropolitana de la Ciudad de México, los cuales no tienen relación aparente con las densidades de las especies. También señala que esta discrepancia, en parte se debe al desconocimiento de los industriales acerca de los procesos óptimos de calentamiento, acordes con las características de las maderas y con la calidad de las chapas.

Efecto del diámetro de las trozas en el
tiempo de acondicionamiento. En el proceso de calentamiento de la madera mediante inmersión en agua, las temperaturas y los tiempos requeridos varían en función de las dimensiones de las trozas o de los cuadrados.

Feihl (1971) indica que el tiempo de calentamiento aumenta aproximadamente con el cuadrado del diámetro y establece que si una troza de diámetro D1 requiere T1 horas para calentarse, el tiempo de calentamiento T2 de una troza de diámetro D2 será de T1x(D2/D1)2. Posteriormente, Feihl y Godin (1975), establecen como regla general, que el tiempo de calentamiento aumenta 4 veces cuando el diámetro de las trozas se duplica $(2 \times 2=4)$ y nueve veces cuando el diámetro se triplica $(3 \times 3=9)$ y así sucesivamente; i.e. si una troza de $30 \mathrm{~cm}(12 \mathrm{plg})$ requiere 10 $\mathrm{h}$; una de $46 \mathrm{~cm}$ (18 plg) requiere $22 \mathrm{1} / 2 \mathrm{~h}$; una de $60 \mathrm{~cm}$ (24 plg) requiere de $40 \mathrm{~h}$; una de $76 \mathrm{~cm}$ (30 plg) requiere de $62 \mathrm{~h}$, etc. Debido a esta diferencia en los requerimientos de tiempo de calentamiento, sugieren que las trozas se segreguen en dos o tres grupos en función del diámetro.

En general Baldwin (1995), Peters (1974), Lutz (1974, 1978) y Moreno y Devlieger (s/f), establecen una relación directa de diámetros de las trozas con los tiempos de calentamiento, basados en experimentos con diferentes especies, sin 
llegar a establecer una regla tan específica como en los dos casos anteriores.

\section{MATERIALES}

Este trabajo se realizó con el apoyo y colaboración de la Empresa ASan Juan Nuevo@que proporcionó la trocería de las especies seleccionadas y de la fábrica de AChapas los Reyes@ que facilitó el aserradero, el equipo para el proceso de calentamiento de la madera, la rebanadora para la obtención de chapa y la estufa para el secado de la chapa.

Tanque de acondicionamiento. El proceso de calentamiento de la madera se realizó en un tanque metálico, con dimensiones de $3.05 \mathrm{~m} \times 1.85 \mathrm{~m}$ y una altura de $1.40 \mathrm{~m}$.

Para el sistema de calentamiento del agua se instaló en el fondo del tanque un serpentín radiador de calor de $4.0 \mathrm{~cm}$ de diámetro. Como medio termoconductor del serpentín se utilizó aceite térmico, calentado por la caldera general de la fábrica. Para proteger al serpentín se instaló una parrilla metálica, sobre la cual se colocaron tres polines de madera que sirvieron de apoyo a los cuadrados 0 flitches de ensayo.

Especies estudiadas. En la selección de las especies estudiadas participó el CIRPAC de Uruapan, Mich. y la propia empresa de ASan Juan Nuevo@tomando como criterio la conformación del arbolado y la abundancia de las especies. Para este estudio se utilizaron dos trozas de cada una de las siguientes especies: $Q$. candicans, $Q$. laurina y $Q$. rugosa.

Termómetro eléctrico. Para el registro de las temperaturas del agua y de la madera durante el proceso de calentamiento de los flitches, se utilizó un termómetro eléctrico con siete canales para la instalación de termopares.
Los termopares están constituidos de dos alambres flexibles de diferentes metales (cobre y hierro) unidos en los extremos (polos $+y-$-) para formar el punto sensor térmico, por el que se capta una corriente eléctrica generada por el calor que activa el sensor térmico. La corriente eléctrica se convierte de análoga a digital, para interpretarse en forma numérica en unidades de temperatura a través del termómetro.

$\begin{array}{llr}\text { Taladro eléctrico, pasta } & \\ \text { impermeabilizadora y agua de }\end{array}$ acondicionamiento. Se utilizó un taladro eléctrico y una broca de $1.0 \mathrm{~cm}$ de diámetro para perforar los cuadrados e insertar los sensores térmicos de los termopares en dichas perforaciones, con la finalidad de medir los cambios de temperatura en la madera, precisamente en el interior de los cuadrados.

Para impermeabilizar los sensores térmicos de los termopares del posible contacto con el agua, se selló con plastilina epóxica la periferia de los orificios en los que se insertaron los sensores. Con esta impermeabilización se evitó la interferencia del agua de calentamiento en la determinación correcta de la temperatura de la madera. Como elemento termoconductor de calor a los cuadrados de madera, se utilizó agua de la red general de distribución, con un $\mathrm{pH}$ de 7.5.

\section{METODOLOGIA}

El proceso de calentamiento de la madera fue similar al que se sigue en la fábrica donde se realizó el estudio, pero con las variantes en cuanto a tiempos de acondicionamiento y temperaturas del agua utilizada, con base en los objetivos del trabajo.

$\begin{array}{llr}\text { Preparación de los cuadrados } & \text { e } \\ \text { instalación de termopares. Para la } & \text { la } \\ \text { evaluación del proceso } & \text { de }\end{array}$


acondicionamiento se utilizaron dos trozas por especie, con longitud promedio de 2.65 $\mathrm{m}$, las cuales se prepararon en el aserradero de la empresa para generar los cuadrados o flitches.

Para la instalación de los termopares en los cuadrados, se realizaron dos perforaciones perpendiculares a la longitud de las trozas, a dos diferentes profundidades en la sección central para ubicar un termopar en el centro del cuadrado y el otro en el punto medio entre dicho centro y la superficie del cuadrado, con separación de $15.0 \mathrm{~cm}$ entre las dos perforaciones.

En cada orificio se insertó un termopar, colocando el extremo sensor en contacto directo con la madera en el fondo del orificio y posteriormente se cubrió la periferia de cada orificio con plastilina epóxica para impermeabilizar al termopar y evitar que el posible contacto con el agua alterara la temperatura real de la madera (Fig.1).
Determinación de la transferencia de calor en los cuadrados. Los cuadrados se colocaron sobre los polines de madera para evitar el calor directo de los serpentines de calentamiento instalados en el fondo del tanque y para ubicarlos en la parte media de la masa de agua. El temopar destinado a medir la temperatura del agua se colocó en la región central de la masa de agua.

El tiempo de calentamiento de los cuadrados se determinó por la temperatura del interior de la madera, considerando un rango de 60 a $70^{\circ} \mathrm{C}$ para especies de alta densidad, como los encinos.

Se registraron las temperaturas del agua y del interior de la madera a intervalos regulares de tiempo, excepto en los periodos nocturnos (10 pm - $6 \mathrm{am}$ ), mediante el termómetro eléctrico descrito. El registro de las temperaturas indicadas por el termómetro, se realizó en forma manual, a intervalos de 30, 60 y 90

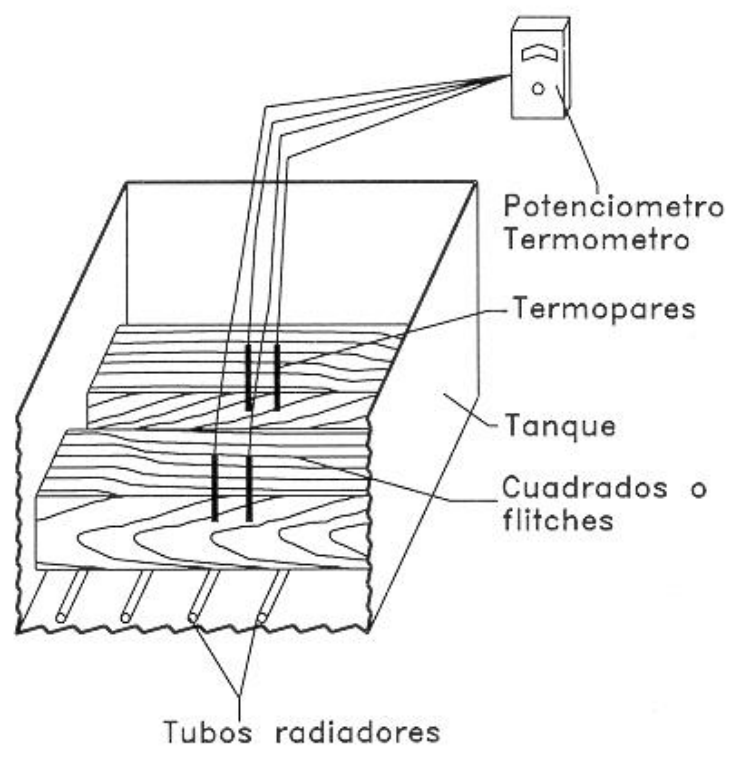

Figura 1. Proceso de calentamiento de la madera y ubicación de termopares 
minutos o más, dependiendo de la etapa de las secuelas de calentamiento, realizándose con mayor frecuencia al inicio del acondicionamiento.

Determinación de la temperatura y calidad de la chapa. Los cuadrados acondicionados se procesaron en una rebanadora vertical para generar las chapas correspondientes de $1 \mathrm{~mm}$ de espesor, los primeros dos cuadrados correspondientes a $Q$. candicans y $Q$. laurina, se procesaron cuando la temperatura interna alcanzó los $60^{\circ} \mathrm{C}$, los de $Q$. candicans y $Q$. rugosa a $65^{\circ} \mathrm{C}$ y los dos últimos de $Q$. laurina y $Q$. rugosa a $70^{\circ}$ C. La chapa generada de cada cuadrado se evaluó en forma visual, con base en su textura, rajaduras y vellosidades. Las calidades de las chapas se clasificaron en tres categorías: A @buena, chapa libre de defectos; AB@ regular, chapa con asperezas, vellosidades y rajaduras hasta en $20 \%$ de la superficie y la categoría $A C @$ mala, a la chapa con los defectos mencionados en una superficie mayor de $20 \%$. Esta clasificación fue exclusiva para este estudio. Además de la calidad del rebanado, la chapa también se evaluó en función del veteado de la madera o arreglo de los elementos constitutivos de la misma.

Secado de la chapa. La chapa generada de cada cuadrado se secó en una estufa progresiva de un nivel, a un contenido de humedad de 5 a $7 \%$, en un tiempo de 22 minutos. Aún cuando se detectó cierto efecto del proceso de secado en la calidad de la chapa (un poco de ondulaciones), no se consideró su evaluación en la calidad de la chapa, porque su efecto es mínimo en la elaboración de madera contrachapada y prácticamente desaparece con el prensado.

\section{RESULTADOS Y DISCUSION}

La información recopilada de los cuadrados procesados de cada una de las tres especies estudiadas se graficó, relacionando el desarrollo de temperaturas del agua y de las dos partes internas de los cuadrados, con el tiempo de calentamiento (Fig. 2).

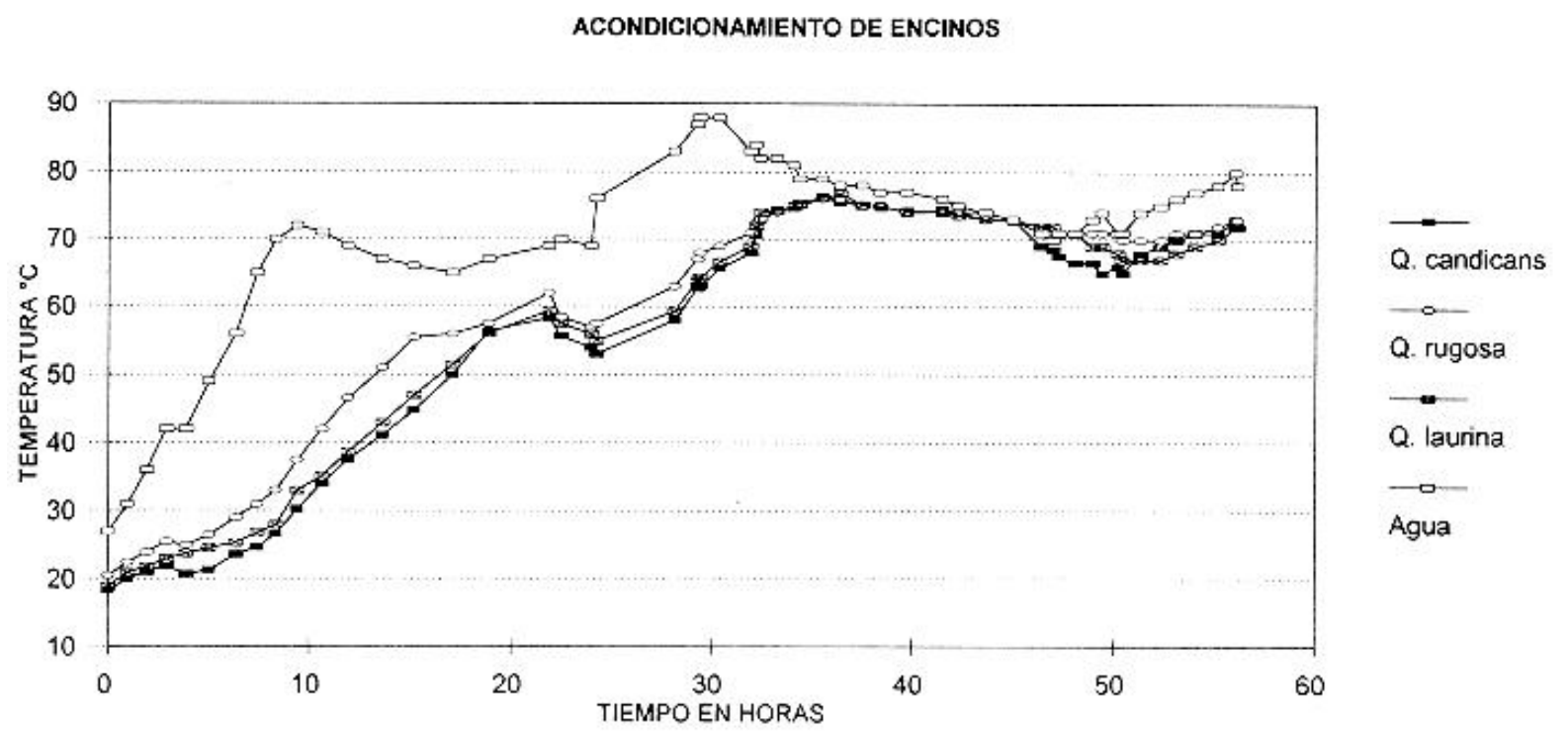

Figura 2. Distribución de la temperatura durante el tiempo de calentamiento de la madera 
Las temperaturas representan los promedios de lecturas internas y externas de los dos cuadrados por especie y la temperatura del agua durante el proceso de calentamiento.La fluctuación de las temperaturas indicadas en la figura 2 , se debe a la forma como se desarrolló el proceso de calentamiento de los cuadrados. El funcionamiento de la caldera general de la fábrica se suspende al término de la jornada laboral (10 pm - 6 am), presentándose periodos de tiempo prolongados con descenso continuo en la temperatura del agua.

Integración de la información del proceso de calentamiento. En la Tabla 1, se integró el tiempo de calentamiento del agua, que comprende la suma de los periodos de tiempo en los que el aceite térmico circuló por el serpentín del tanque para calentar el agua; el tiempo de acondicionamiento, que es el tiempo total de permanencia del cuadrado en el agua del tanque; la temperatura máxima interna, el centro del cuadrado y con éstas se generó la temperatura final promedio del interior del cuadrado y la temperatura de corte que se refiere a la temperatura de la madera al momento de rebanar el cuadrado.
En general, se considera que los encinos se pueden procesar para la producción de chapa rebanada en un rango de temperatura interna del flitch de 60 a $75^{\circ} \mathrm{C}$. El tiempo requerido para alcanzar esta temperatura varía con el medio de calentamiento (vapor, agua, aceite térmico) y con las prácticas donde se efectúa este proceso. Aún cuando es común en la industria de madera contrachapada en México manejar tiempos definidos de calentamiento, este parámetro puede variar entre empresas, puesto que las prácticas de acondicionamiento cambian en función de las temperaturas del medio transmisor, de los periodos en que se operen los sistemas de calentamiento (en periodos continuos de $24 \mathrm{~h}$ diarias o únicamente en los turnos diurnos) y de las condiciones de las pilas de acondicionamiento, por lo que se considera inapropiado señalar un lapso definido, como se acostumbra en la industria de madera contrachapada. Sin embargo, se sugiere que se tomen como índices los tiempos señalados en este trabajo y como referencia principal la temperatura de los cuadrados al momento de sacarlos de las pilas de calentamiento o de la chapa al momento de efectuar el rebanado de los cuadrados. En relación a

TABLA 1. Temperaturas y tiempo de calentamiento de los cuadrados de madera de encino

\begin{tabular}{||l|c|c|c|c|c|c|c|c||}
\hline \hline ESPECIE & FLITCH & $\begin{array}{c}\text { DIMENS. } \\
\text { TRANSV. } \\
\text { FLITCH } \\
\mathrm{cm}\end{array}$ & $\begin{array}{c}\text { TIEMPO } \\
\text { CALENT. } \\
\text { AGUA } \\
\mathrm{h}\end{array}$ & $\begin{array}{c}\text { TIEMPO } \\
\text { DE } \\
\text { ACOND. } \\
\mathrm{h}\end{array}$ & $\begin{array}{c}\text { TEMP. } \\
\text { MAXIMA } \\
\text { INTERNA } \\
{ }^{\circ} \mathrm{C}\end{array}$ & $\begin{array}{c}\text { TEMP. } \\
\text { FINAL } \\
\text { PROM. } \\
{ }^{\circ} \mathrm{C}\end{array}$ & $\begin{array}{c}\text { TEMP. } \\
\text { DE } \\
\text { CORTE } \\
{ }^{\circ} \mathrm{C}\end{array}$ & $\begin{array}{c}\text { CALIDAD } \\
\text { DE LA } \\
\text { CHAPA } \\
\text { categoría }\end{array}$ \\
\hline Q. candicans & $\mathrm{a}$ & $48 \times 25$ & 29.2 & 54.1 & 62.0 & 61.0 & 54.0 & buena \\
b. laurina & $\mathrm{b}$ & $42 \times 23$ & 34.2 & 58.2 & 66.0 & 62.0 & 56.0 & buena \\
\hline Q. rugosa & $\mathrm{a}$ & $56 \times 32$ & 36.2 & 58.3 & 63.0 & 61.0 & 50.0 & buena \\
& $\mathrm{b}$ & $46 \times 28$ & 40.0 & 62.3 & 72.0 & 71.0 & 55.0 & buena \\
\hline & $\mathrm{a}$ & $47 \times 36$ & 35.1 & 52.3 & 67.0 & 65.0 & 51.0 & $\begin{array}{c}\text { regular } \\
\text { regular }\end{array}$ \\
\hline
\end{tabular}


la calidad de la chapa por tipo de especie, la de $Q$. candicans y $Q$. laurina generaron la mejor calidad en cuanto a textura, desarrollo de grietas y vellosidades, al momento de efectuar el rebanado $y$ después del proceso de secado de la chapa. Q. rugosa fue la especie que generó una chapa mas áspera, básicamente por la presencia de rayos mas grandes, que tienden a provocar orificios en la chapa, además de ser bastante notoria la presencia de manchas café oscuro, posiblemente por ataque de bacterias en el arbolado en pie, más que por el proceso de oxidación, que también se puede presentar durante el calentamiento por la presencia de taninos. Se puede considerar que en general la chapa de las especies procesadas presenta un veteado uniforme, aún en $Q$. candicans y $Q$. laurina que son de porosidad circular, no se presentaron las franjas de los poros o las diferencias de la madera de primavera y de verano que le imprimen a la chapa el veteado de Acatedrales@ uno de los atractivos más buscados en los encinos americanos. Esta característica podría ser un factor limitante de estas tres especies para ser utilizadas en la producción de chapa para madera contrachapada, que se podrá contrarrestar paulatinamente con la promoción del uso de los encinos mexicanos para madera contrachapada, con las cualidades intrínsecas de veteado de las especies nacionales.

\section{CONCLUSIONES Y RECOMENDACIONES}

1. Desde el punto de vista de procesos (ablandamiento, rebanado y secado de la chapa), se considera que no existe ningún problema técnico para generar chapa de buena calidad, optimizando la interrelación maderaprocesos.

2. El rango de temperaturas de la madera para optimizar el rebanado de los cuadrados para la producción de chapa oscila entre 60 a $75^{\circ} \mathrm{C}$ en la parte más interna del cuadrado 0 flitch.

3. Debido a que no se tienen bosques cultivados de encinos, aún cuando dos de las especies utilizadas en este estudio son de porosidad circular $(Q$. candicans y $Q$. laurina), esta característica no se manifestó en el veteado de la chapa, lo que puede limitar su utilización en la producción de madera contrachapada, por la preferencia del consumidor al veteado en "catedrales" de los encinos americanos.

4. Por la carencia de veteado de las especies usadas en este estudio, se sugiere adoptar un sistema de comercialización propio para encinos mexicanos, que compita en calidad con la madera contrachapada de encinos americanos.

5. Se sugiere ampliar este tipo de estudios, con otras especies potencialmente aptas para chapa en función de su abundancia y conformación del arbolado y de preferencia con porosidad circular, como $Q$. acutifolia, $Q$. affinis, $Q$. castanea, $Q$. crassifolia, $Q$. crassipes, Q. conspersa, Q. durifolia, Q. excelsa, $Q$. potosina, $Q$. sideroxyla.

6. Por su efecto negativo en los procesos de producción y en la calidad de la chapa, se sugiere no utilizar trozas muy nudosas, ni manchadas, ni podridas, estos dos últimos aspectos se acentúan más en arbolado maduro.

7. Se sugiere utilizar los tiempos de calentamiento de la madera de este estudio como una referencia, considerando que pueden cambiar con las temperaturas usadas en otras fábricas y con los medios 
termoconductores empleados (agua, vapor, aceite térmico).

\section{REFERENCIAS}

Andrade E. y M. Alvarez. 1997. Comunicación personal.

Baldwin, F.R. 1995. Plywood and veneerbased products manufacturing practices. Miller Feeman Publications. EUA. 388 p.

De la Paz P.O.,C. 1976. Características anatómicas de cinco encinos de México. SAG. SFF. INIF. Bol. Tec. No. 46. $43 \mathrm{p}$.

De la Paz P.O.,C. y M. L. Aguilar E. 1978. Diferencias morfológicas externas y anatómicas de la madera de encinos blancos y rojos. SAG. SFF. INIF. Bol. Tec. No. 59. 19 p.

Feihl, O. 1971. Heating frozen and nonfrozen veneer logs. Dep. Env., Can. For. Serv., East. For. Prod. Lab., Canadá. 50 p.

Feihl, O. y V. Godin. 1975. Heating veneer logs. A practical Guide. Can. For. Serv. Technical Report 9. Ottawa, On. Canadá. 20 p.

Fleischer, H.O. 1959. Heating rates for logs, bolts and flitches to be cut into veneer. U.S.D.A., For. Prod. Lab., For. Serv., Div. Tim. Proc., Report. N 2149. Madison, WI. EUA. 18 p.

Lutz, J., F., A. Mergen y H. Pazner. 1967. Effect of moisture content and speed of cut on quality of rotary-cut veneer. USDA. Forest Service. Forest Products Lab. Research Paper FPL0176. Madison, WI. EUA. 13 p.

Lutz, J.F. 1972. Veneer species that grow en the United States. USDA. Forest
Service. FPL. 167. Madison, WI. EUA. $127 \mathrm{p}$.

Lutz, J. F. 1974. Techniques for peeling, slicing, and drying veneer. USDA. Forest Service. Forest Products Lab. Research Paper FPL 228. Madison, WI. EUA. 64 p.

Lutz, J. F. y R.A. Patzer. 1976. Spin-out of Veneer Blocks During Rotary Cutting of Veneer. USDA. Forest Service. Forest Products Lab. Research Paper FPL 276. Madison, WI. EUA. 23 p.

Lutz, J.F. 1978. Wood Veneer: Log Selection, Cutting, and Drying. USDA. Forest Service. Tech. Bull. No. 1577. Madison, WI. EUA. 137 p.

Moreno M.,R. y F. Devlieger S. (s/f) Influencia de los factores de la madera y de las condiciones del macerado en el tiempo de calentamiento de las trozas. Universidad Austral de Chile. Fac. Cien. For. y Fac. Cien. Ingen. Valdivia, Chile. 35 p.

Palka, L.C. 1974. Veneer Cutting Review Factors Affecting and Models Describing the Process. Report. VP-X135 Dep. Env. Can. For. Serv. West. For. Prod. Lab. Canadá. 54 p.

Peters, C.W. 1974. Peeler block preconditioning. Proceedings of the Modern Plywood Techniques Vol. 1. Hubert G. Lambert. Edit. Portl. EUA. $160 \mathrm{p}$.

Walser, D.C. 1974. Steam-Injection Knife Improves Veneer Quality. For. Prod. Journal. Vol. 24, No.9. p:70-79 p.

Voirol, L.A. 1985. El desenrollo del encino; una alternativa de utilización. II Seminario Nacional sobre la Utilización de Encinos. Guadalajara, Jal. 22-24 Mayo, 1985. p:321-328. 
Zavala Z., D. 1991. Diagnóstico de la industria de tableros contrachapados en el área metropolitana del Distrito Federal. Ciencia Forestal Vol. 15. No. 68. Jul.-Dic. 61-83 pp.
Zavala, Z., D. y G. Trujillo A. 1993. Análisis del proceso de calentamiento de trocería para la producción de chapa. Ciencia Forestal 18(74):139-162. 